\title{
NOTE ON A RESULT OF LEVINE AND LIFSCHITZ
}

\section{I. HIRSCHMAN, JR. AND J. A. JENKINS}

In a paper some years ago, [1], ${ }^{1}$ Levine and Lifschitz considered, among other questions, the relationship between the gaps of a Fourier series and its admissible integral orders of zeros, a problem first treated by S. Mandelbrojt [2]. Let $f(t)$ be a function summable over the interval $(-\pi, \pi)$ and let

$$
f(t) \sim \frac{1}{2} a_{0}+\sum_{n=1}^{\infty} a_{n} \cos n t+b_{n} \sin n t
$$

be its associated Fourier series. Let $\psi(\alpha)$ be a continuous non-negative nondecreasing function defined for $0 \leqq \alpha \leqq 2 \pi$. We shall say that $f(t)$ has a left-hand zero of integral order $\psi(\alpha)$ at $t=\pi$ if

$$
\phi(\alpha) \equiv \int_{\pi-\alpha}^{\pi}|f(t)| d t \leqq \psi(\alpha) \quad(0 \leqq \alpha \leqq 2 \pi) .
$$

(One could equally well consider such a zero at any other point.) $\mathrm{Na}$ tural choices for $\psi(\alpha)$ are:
a.
$\psi(\alpha)=\alpha^{n}$
$(n=1,2, \cdots)$,
b.
$\psi(\alpha)=e^{-(1 / \alpha)^{\rho}}$
$(0<\rho<\infty)$,
c.
$\psi(\alpha)\left\{\begin{array}{l}=0 \\ >0\end{array}\right.$
$\left(0 \leqq \alpha \leqq \alpha_{0}\right)$,
$\left(\alpha_{0}<\alpha \leqq 2 \pi\right)$.

If $\psi(\alpha) \not \equiv$, then there exists a number $\alpha_{0}$ such that $\psi(\alpha)=0\left(0 \leqq \alpha \leqq \alpha_{0}\right)$ and $\psi(\alpha)>0\left(\alpha_{0}<\alpha \leqq 2 \pi\right)$. Let

$$
r=-\log \psi(\alpha) / \alpha \quad\left(\alpha_{0}<\alpha \leqq 2 \pi\right) .
$$

It is easily seen that for all $r$ sufficiently large this equation may be inverted to give $\alpha$ as a function $\alpha=\eta(r)$ of $r$. It is $\eta(r)$ which we shall use as the measure of the zero of $f(t)$. It should be noted that such extreme behavior as a. and c. may be permitted and that our theorems are significant throughout this entire range.

Let $N(t)$ be the number of indices $n<t$ whose coefficients $a_{n}$ and $b_{n}$ are not both equal to zero. The density of these indices may be characterized by the function 1949.

Presented to the Society, February 26, 1949; received by the editors February 19,

1 Numbers in brackets refer to the references cited at the end of the paper 


$$
\zeta(r)=\log r+2 r^{2} \int_{0}^{\infty} \frac{N(t)}{t\left(t^{2}+r^{2}\right)} d t .
$$

This slight correction to the original formula of Levine and Lifschitz was pointed out by $S$. Mandelbrojt in his review of their paper, see [3].

Levine and Lifschitz proved that if

$$
\lim _{r \rightarrow \infty} \inf [3 \zeta(2 r)-r \eta(r)]=-\infty,
$$

then $f(t)=0$ almost everywhere in $(-\pi, \pi)$. In particular

$$
\liminf _{r \rightarrow \infty}[\operatorname{c\zeta }(r)-r \eta(r)]=-\infty
$$

implies $f(x)=0$ almost everywhere if $c=12$, since $\zeta(\alpha r) \leqq \alpha^{2} \zeta(r), \alpha>1$, $r$ sufficiently large. In the other direction Levine and Lifschitz proved that this does not hold for $c<1 / 2$. It is the object of this note to show that it does hold for $c=\pi$.

The method of proof is to construct the function

$$
Q(z)=z \prod_{k=1}^{\infty}\left(1-\frac{z^{2}}{n_{k}^{2}}\right)
$$

where $n_{k}$ are the values of the indices of the nonzero coefficients in the series (1). $Q(z)$ assumes its maximum modulus for a circle with center the origin on the imaginary axis and

$$
\log |Q(i y)|=\zeta(y)
$$

The integral function

$$
F(z)=\frac{1}{\pi} \int_{-\pi}^{\pi} f(t) e^{-i t z} d t
$$

has zeros at those integers where $Q(z)$ does not. Thus it is easily proved that the function

$$
\Phi(z)=\frac{F(z) Q(z)}{\sin \pi z}
$$

is integral and satisfies the bound

$$
|\Phi(z)|<D e^{s(|z|)}
$$

for all $z$, and for $y>0$ the bound

$$
|\Phi(z)|<D e^{\zeta(|z|)-y y(y)} \quad\left(z=x+i y=r e^{i \theta}\right) .
$$


For these results we refer to [1].

Suppose that $\Phi(z) \not \equiv 0$; then $z=0$ is a zero of some finite order $m, 0 \leqq m<\infty$. Let us regard the circle $C:|z|=r$. On this circle

$$
\begin{array}{rlr}
\log \left|\Phi(z) / D z^{m}\right| & \leqq \zeta(r)-m \log r & (\pi \leqq \arg z \leqq 2 \pi) \\
& \leqq \zeta(r)-m \log r-r \sin \theta \eta(r \sin \theta) \quad(0 \leqq \arg z \leqq \pi) \\
& \leqq \zeta(r)-m \log r-\sin \theta r \eta(r), &
\end{array}
$$

since $\eta(r)$ is a nonincreasing function of $r$. Because the harmonic measure at the origin of an infinitesimal arc of length $r d \theta$ on the circle $C$ is $(1 / 2 \pi) d \theta$, we have, by an integrated form of the two constants theorem,

$$
\begin{aligned}
\log \left|\Phi(z) / D z^{m}\right|_{z=1} & \leqq \frac{1}{2 \pi} \int_{0}^{2 \pi}[\zeta(r)-m \log r] d \theta-\frac{1}{2 \pi} \int_{0}^{\pi} r \eta(r) \sin \theta d \theta \\
& \leqq \frac{1}{\pi}[\pi \zeta(r)-r \eta(r)-\pi m \log r] .
\end{aligned}
$$

Because we have assumed

$$
\liminf _{r \rightarrow \infty}[\pi \zeta(r)-r \eta(r)]=-\infty,
$$

it follows that $\left[\Phi(z) / z^{m}\right]_{z-0}=0$, a contradiction. Thus we must have $\Phi(z) \equiv 0$. Consequently $F(z) \equiv 0$ and $f(t)=0$ almost everywhere in the interval $(-\pi, \pi)$.

$f(t)$ is said to have a two-sided zero of integral order $\psi(\alpha)$ at $t=\pi$ if

$$
\phi(\alpha)=\int_{-\pi}^{-\pi+\alpha}|f(t)| d t+\int_{\pi-\alpha}^{\pi}|f(t)| d t \leqq \psi(\alpha) \quad(0 \leqq \alpha \leqq \pi) .
$$

In this case a simple adaptation of the above argument shows that the condition

$$
\liminf _{r \rightarrow \infty}\left[\frac{\pi}{2} \zeta(r)-r \eta(r)\right]=-\infty
$$

is sufficient in order to have $f(t)=0$ almost everywhere in $(-\pi, \pi)$.

If in the above conditions we replace lim inf by lim, more precise results may be obtained. Indeed if $f(t)$ has a two-sided zero at $t=\pi$, the condition

$$
\lim _{r \rightarrow \infty}[(1+\epsilon) \zeta(r)-r \eta(r)]=-\infty
$$

implies that $f(t)=0$ almost everywhere in $(-\pi, \pi)$. This follows 
from the fact that on the lines $\arg z=\pi / 2+\delta$, $\arg z=-\pi / 2-\delta$, we have the bound

$$
|\Phi(z)| \leqq D e^{5(|z|)-|z| \cos \delta \eta n(|z| \cos \delta)} \leqq D e^{\zeta(|z|)-\cos 8|z| \eta(|z|)}
$$

so that if $\cos \delta \geqq(1+\epsilon)^{-1}, \Phi(z)$ tends to 0 as we go to infinity on these lines. Then, since $\Phi(z)$ is at most of order one, by the PhragmenLindelöf Principle it is bounded in the enclosed angle and this follows similarly for the angle $-\pi / 2+\delta \leqq \arg z \leqq \pi / 2-\delta$ and the two complementary angles. Thus $\Phi(z) \equiv 0$ and the result is proved.

If $f(t)$ has a one-sided zero at $t=\pi$, the same argument applied to $\Phi(z) \Phi(-z)$ shows that the condition

$$
\lim _{r \rightarrow \infty}[(2+\epsilon) \zeta(r)-r \eta(r)]=-\infty
$$

implies that $f(t)=0$ almost everywhere in $(-\pi, \pi)$.

It is easy to give examples which provide lower bounds for the constant $c$ which may occur in the above result. Indeed, let $f_{1}(t)$ be identically zero from $-\pi+\delta$ to $\pi$ and arbitrary (but not zero) in the rest of $(-\pi, \pi)$. We see at once that $\zeta(r)$ is asymptotically not greater than $\pi r$ while for the choice $\psi(\alpha)=\phi(\alpha), r \eta(r)$ is asymptotically equal to $(2 \pi-\delta) r$. Thus the constant $c$ cannot be taken less than 2. Similarly the function $f_{2}(t)$ identically zero outside $(-\delta, \delta)$, arbitrary in this interval, provides an example showing that for a two-sided zero the constant $c$ cannot be taken as less than 1 .

It should be mentioned that this does not, of course, supersede the result of Levine and Lifschitz since they showed that $c$ cannot be taken less than $1 / 2$ even when only very restricted classes of lacunary series are admitted.

\section{REFERENCES}

1. B. Levine and M. Lifschitz, Quasi-analytic functions represented by Fourier series, Rec. Math. (Mat. Sbornik) N.S. vol. 9 (1941) pp. 693-711.

2. S. Mandelbrojt, Series de Fourier et classes quasianalytiques de fonctions, Paris, 1935.

3. - Review of [1], Mathematical Reviews vol. 3 (1942) p. 106.

HARVARD UNIVERSITY 